\title{
Abrupt onsets capture attention independent of top-down control settings II: Additivity is no evidence for filtering
}

\author{
Daniel SchreiJ, Jan Theeuwes, ANd Christian N. L. Olivers \\ Vrije Universiteit Amsterdam, Amsterdam, The Netherlands
}

\begin{abstract}
Is attentional capture contingent on top-down control settings or involuntarily driven by salient stimuli? Supporting the stimulus-driven attentional capture view, Schreij, Owens, and Theeuwes (2008) found that an onset distractor caused a response delay, in spite of participants' having adopted an attentional set for a color feature. However, Folk, Remington, and Wu (2009) claimed that this delay reflects separate, nonspatial filtering costs instead, because the onset effects were additive with color-based capture effects, and capture should have caused underadditivity. The present Experiment 1 shows that contingent capture caused by additional color cues is also additive, just like the onset effect. This makes additivity a dubious diagnostic with regard to spatial capture. Experiment 2 demonstrates that it is possible to obtain underadditivity when attention-demanding distractors have sufficient capturing power. Experiment 3 shows that the abrupt onset interference turns into a benefit when the locations of the onset and the target coincide. Together, these results argue in favor of stimulus-driven attentional capture by abrupt onsets.
\end{abstract}

A frequently debated issue in attention research is which underlying cognitive mechanisms are fundamental for the ability of objects to capture attention. Can attentional capture be driven by bottom-up, stimulus-related factors, or is capture always contingent on what the observer is looking for (i.e., his or her top-down attentional set)? The latter stance has been advocated by Folk, Remington, and colleagues on the basis of spatial cuing studies in which observers looked, for example, for a red target among white distractors (Folk \& Remington, 1998; Folk, Remington, \& Johnston, 1992). Prior to the target display, a cue appeared that unpredictably indicated the target position (valid cue) or one of the distractor positions (invalid cue). Even though the cues were uninformative, valid cues resulted in faster response times (RTs), but only when the cue matched the target-defining property - that is, when it was also red. When the cue was defined instead by a single abrupt onset, there was no cuing effect. This suggests that the cue captures attention in a spatial manner, but only when it carries a feature relevant to the goals of the observer. In other words, attentional capture is contingent on the top-down attentional set (but for a different account of contingent capture results, see Belopolsky, Schreij, \& Theeuwes, 2010).

Recently, Schreij, Owens, and Theeuwes (2008) reported evidence that appears to be inconsistent with the contingent capture hypothesis. In a task very similar to the spatial cuing paradigm of Folk et al. (1992), Schreij et al. found that, even when observers had an attentional set for color, the presence of an irrelevant abrupt onset nevertheless slowed participants in finding the target. As in the Folk et al. (1992) paradigm, participants searched for a red target, and an attentional set for redness was indeed demonstrated by a strong validity effect of the matching red precue. At the same time, the presence of an abrupt onset in the target display interfered with responding, whether the red cue was valid or invalid. Consistent with earlier claims suggesting that onsets capture attention in a stimulus-driven manner (Belopolsky, Theeuwes, \& Kramer, 2005; Christ \& Abrams, 2008; Enns, Austen, Di Lollo, Rauschenberger, \& Yantis, 2001; Gellatly, 1999; Remington, Johnston, \& Yantis, 1986; Theeuwes, 1990, 1994; Yantis \& Jonides, 1984), Schreij et al. concluded that even when observers adopt a clear top-down set for color, they cannot prevent attentional capture by the onset. According to the contingent capture hypothesis, the presence of the onset should have had no effect on responding, because it was completely irrelevant to the task.

However, Folk, Remington, and Wu (2009) have questioned whether the interference caused by the abrupt onset distractor was due to a bottom-up shift of attention to the location of the onset. In line with the earlier claims of Folk and Remington (1998), they argued that the interference was due to nonspatial filtering costs, a notion, first introduced by Kahneman, Treisman, and Burkell (1983), according to which irrelevant new objects that appear simultaneously with the target compete for attention and need to be filtered out. This filtering operation slows RTs to the target; in other words, an irrelevant object can cause a delay in the deployment of attention to a

D. Schreij, dbb.schreij@psy.vu.nl 
relevant object without itself invoking a shift of attention. Specifically, in the Schreij et al. (2008) study, the onset would be competing with the target, thus causing filtering costs. The filtering explanation is compatible with the contingent capture hypothesis because attention goes only to the item that matches the attentional set for color; this explanation assumes that attention does not go to the location of the abrupt onset, which causes only a nonspecific filtering cost. Because capture and filtering operations are presumed to take place during independent stages of processing, additive effects of color-based cuing and onset interference would be expected (Sternberg, 1969). As pointed out by Folk et al. (2009), this was exactly what was found by Schreij et al.: The interference caused by the onset presence was equally strong for trials with valid color cues and for trials with invalid ones. Therefore, if one accepts that the color cue captures spatial attention, the onset cannot also do so. According to Folk et al. (2009), if the onset presence and color-based cuing both operated on the same process of spatial capture, one would expect these two factors to show an underadditive relationship instead. After all, if one assumes that capture by the onset made the cue obsolete, attention should move directly from onset to target, regardless of cue validity. Thus, according to Folk et al. (2009), the elimination of attentional capture is instantaneous, in that the subsequent appearance of another salient object "should effectively eliminate the [earlier] effects" (p. 309).

However, we assume that the local activation of an object does not have to be instantaneous. Activity takes some time to build up, and may take even more time to dissipate. Especially when a distractor object looks like a target object, resource allocation may be more sustained, making it difficult to disengage from this object (see Theeuwes, Atchley, \& Kramer, 2000). If one accepts that an object's capturing power can be sustained for a while (especially when it looks task relevant), the additivity of color-based cuing and onset interference is not that strange from an attentional capture perspective. The abrupt onset may briefly capture attention away from the red cue; however, because the onset is not relevant but the red cue is, attention rapidly returns to the latter. An alternative solution is that attention first lingers for quite some time at the cued location, even after the cue has disappeared and the target display is already present. Thus, attention can already gather some evidence about whatever object (possibly a target) is present at that location. This process is then disrupted, but not reset, because attention is captured away by the abrupt onset. When attention in turn disengages from the abrupt onset, the color cues may indeed already be forgotten, as Folk et al. (2009) suggested, and attention moves directly back to the target. However, in the case of a valid cue, a large part of the evidence on which response should be made might have already been accumulated by then, resulting in a speeded response (i.e., a cuing effect that is additive with an onset effect).

This article investigates the claim that two objects that subsequently capture attention should always show an underadditive relationship. If Folk et al. (2009) are correct that capture by a subsequent object always nullifies any previous capture effects, such underadditivity should also be found when both consecutive capturing objects are contingent on the observer's attentional set. However, if additivity is found in this case as well, the criterion of underadditivity cannot be regarded as a suitable diagnostic for or against the occurrence of attentional capture.

Using further adaptations of the Folk et al. (1992) paradigm, Experiment 1 demonstrates that interference by a distractor is also additive with the cuing effect when the distractor carries the task-relevant feature and thus, according to the theory of contingent attention capture, is assumed to capture attention. Experiment 2 shows that a dis-

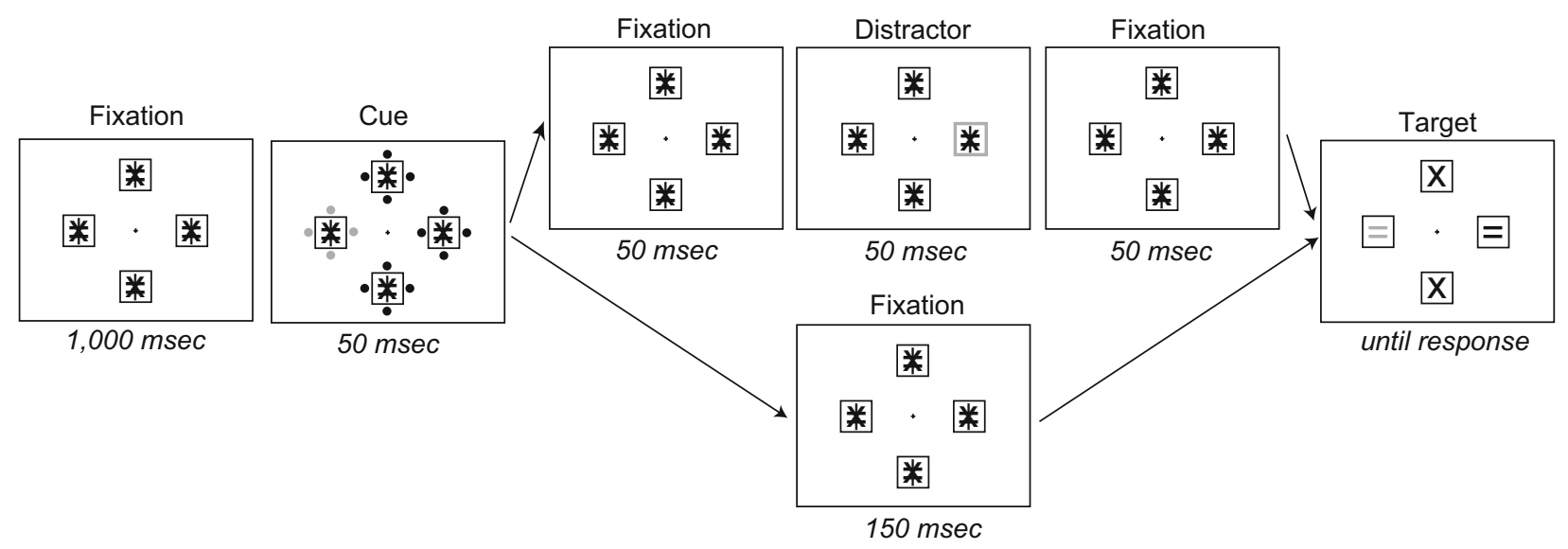

Figure 1. An illustration of the sequence of events for a trial of Experiment 1. First, a fixation display is shown for $\mathbf{5 0 0}$ msec, after which the central fixation cross is turned off for $50 \mathrm{msec}$. After this, the fixation display is shown again for a period of $1,000 \mathrm{msec}$. Then a cue display appears for $50 \mathrm{msec}$, and, after an interstimulus interval of $150 \mathrm{msec}$ in which the fixation display is shown again, the target display is presented for $\mathbf{2 , 0 0 0}$ msec or until the participant responds. When there was a distractor cue, it was presented for 50 msec between the cue and target display and was preceded and succeeded by a 50-msec fixation display. This is an example of a valid trial, since the location of the red balls in the cue display and the location of the red letter in the target display are the same. In reality the background was black, black lines where white, and the gray elements were red. 
tractor is actually able to completely eliminate the effects of a precue, resulting in an underadditive relation, when it both has a strong bottom-up signal and is contingent on the participant's attentional set. As these experiments show, the occurrence of additivity is not a reliable diagnostic for or against attentional capture, but depends on the relative strength of the distractor. Experiment 3 shows that the additional abrupt onset yielded considerable RT benefits, rather than costs, when its location incidentally coincided with the target. This too indicates that the new abrupt onset element actually attracted attention to its location, rather than resulting in general filtering costs.

\section{EXPERIMENT 1}

This experiment investigated whether a pattern of additivity can serve as a useful diagnostic against attentional capture. We took the Schreij et al. (2008) version of the Folk et al. (1992) paradigm, but we left out the onset distractor and instead briefly made one of the other distractor boxes red. This red distractor was always invalid. Thus, the displays contained a red cue (valid or invalid), then, potentially, a red distractor (invalid), and finally, the target display. The contingent capture theory states that the new red distractor should capture attention away from the initially cued location; after all, red is what the observers are looking for, and capture by red is what explains the original cuing effect. If such capture by a new distractor did indeed erase or reduce all prior cuing effects, the red distractor should attenuate here the benefits of a valid color cue, resulting in underadditivity. If, on the other hand, there is still residual activation of the first cue strong enough to affect the reorienting toward or the identification of the target, we may again observe additivity between cue validity and the presence of the red distractor.

To further support the claim that both cues captured attention, we investigated whether cued distractor identities interfered with responses to the target, following the identity intrusion method introduced by Theeuwes (1996; see also Theeuwes \& Burger, 1998). If attention is shifted to the location of the cue, the assumption is that the identity of the object at that position will be preferentially processed (e.g., Kramer \& Jacobson, 1991). If the distractor identity is compatible with the target identity, performance may benefit, relative to when distractor and target are incompatible. This compatibility effect would further strengthen the claim that the distractor captured spatial attention (see Folk, Leber, \& Egeth, 2002, for a similar argument).

\section{Method}

Participants. Ten students from the Vrije Universiteit Amsterdam, age range 18-27 (average 21), participated in this experiment in return for money or course credits. All reported no color blindness and normal or corrected-to-normal vision.

Apparatus and Stimuli. The experiment was run on an HP Compaq with a 2.6-GHz Pentium 4 processor and $512 \mathrm{MB}$ of RAM. The stimuli were presented on a 19-in. Iiyama Vision Master Pro 454 CRT screen with loudspeakers, with a refresh rate of $120 \mathrm{~Hz}$ and a resolution of $1,024 \times 768$ pixels. The " $\mathrm{X}$ " and " $\mathrm{M}$ " keys on a normal keyboard were used to register the participants' responses. Stimu- lus presentation and response recording were done in E-Prime 1.2 (Psychology Software Tools, 2003). The experiment was executed in a dimly lit, soundproofed room, in which participants were seated approximately $75 \mathrm{~cm}$ from the screen. All displays had a uniform black background. The fixation display consisted of a bright white $\left[\operatorname{CIE}(0.286,0.311), 59,50 \mathrm{~cd} / \mathrm{m}^{2}\right]$ fixation cross at the center of the screen, surrounded by four light gray $[\operatorname{CIE}(0.285,0.306)$, luminance $28.28 \mathrm{~cd} / \mathrm{m}^{2}$ ] placeholder boxes measuring a width of $3.4^{\circ}$ visual angle. The four boxes were positioned above, below, to the left, and to the right of the fixation cross, along a virtual circle with a radius of $6.6^{\circ}$ visual angle, with the fixation cross at the center. The cue display consisted of the same elements as the fixation display, with the addition of four dots, with a diameter of $0.5^{\circ}$ visual angle, positioned along the outside of the center of each rib of all the placeholder boxes. One set of these dots surrounding one of the placeholder boxes were in red $\left[\operatorname{CIE}(0.621,0.345), 10.43 \mathrm{~cd} / \mathrm{m}^{2}\right]$ and indicated the cued location. All the dots surrounding the other boxes were bright white $\left[\operatorname{CIE}(0.286,0.311), 59.50 \mathrm{~cd} / \mathrm{m}^{2}\right]$. Until the presentation of the target screen, each of the boxes contained a bright white figure consisting of overlapping "X," "|," and "=" symbols. When the target display was presented, the irrelevant line segments were removed, revealing an "X" (21-pt Myriad Roman) or an "=" (22-pt Myriad Roman bold) inside each box. At the same moment, the color of the target character turned from white to red. There were always two "X"s and two "="s present. To serve as a distractor cue, one of the placeholder boxes briefly flashed from white to red [CIE $(0.621$, $\left.0.345), 10.43 \mathrm{~cd} / \mathrm{m}^{2}\right]$.

Design and Procedure. There were two important factors. The first was the validity of the first color cue, which could be valid or invalid. The second was the presence of a red distractor (absent or present). This resulted in a $2 \times 2$ factorial design. The red distractor was always invalid and would never appear on a cued location, whereas the first color cue was valid in only $25 \%$ of the trials and invalid in the rest. The first cue validity was varied within blocks, and the presence of the second cue was varied between blocks. There were nine blocks of 80 trials, of which the first block was a practice block.

Participants were tested in a 30-min session and were given oral instructions to familiarize them with the task before the experiment started. They were told to keep an index finger on each of the two response buttons and to not move their eyes away from fixation during a trial, because this would impair their performance. The target character, equally often an "X" or an "=," randomly mixed within blocks, appeared in one of the present boxes on the display. At the end of each block, participants were advised to take a rest and were told to wait for at least $30 \mathrm{sec}$ before they could continue.

Trials began with the presentation of the fixation display for $1,000 \mathrm{msec}$, after which the fixation cross blinked off and on for $100 \mathrm{msec}$, to notify the participant of the start of a trial. The fixation display then remained on the screen for another $100 \mathrm{msec}$, after which a cue display was presented for $50 \mathrm{msec}$. If a distractor cue was present, it appeared $50 \mathrm{msec}$ after the first cue disappeared, with a duration of $50 \mathrm{msec}$. Afterward, the fixation display was again presented for $50 \mathrm{msec}$ before the target display appeared. If the second cue was absent, the first cue would simply be followed by a fixation display for $150 \mathrm{msec}$, after which the search display was presented until the participant responded (for a maximum of 2,000 msec). The participant was instructed to look for the red item and press the " $\mathrm{X}$ " key when it was an X, or press the "M" key when it was an $=$. Distinctive sounds were played for correct or incorrect responses. If the response was incorrect, the experiment paused for $5 \mathrm{sec}$ to let the participants regain their focus. There was an intertrial interval of $500 \mathrm{msec}$. (See Figure 1 for an illustration of the event sequence in an Experiment 1 trial.)

\section{Results and Discussion}

Incorrect responses were removed from the data set, resulting in a loss of $3.3 \%$ of the trials; responses with RTs 
below and above $2.5 S D$ (another $2 \%$ ) were also removed. The remaining data, shown in Figure 2, were first submitted to an ANOVA with red distractor (absent, present) and cue validity (valid, invalid) as factors.

There was a significant main effect of cue validity $[F(1,9)=19.77, p<.001]$. Participants were slower after an invalid cue than after a valid one. The presence of the red distractor slowed the responses by $18 \mathrm{msec}$ [red distractor, $F(1,9)=13.76, p<.001]$. There was no interaction between distractor cue and cue validity $[F(1,9)<0.5$, $p>.5]$, indicating additive effects of distractor presence and cue validity. The same analysis of the error pattern revealed no significant effects $[F(1,9)=0.882, p=.372]$.

Another analysis investigated possible compatibility effects between the target character and the characters located at the color cue and the red distractor. When there was no red distractor present and the cue was invalid, the character at the cued location showed a reliable compatibility effect with the target $[t(9)=2.61, p<.05]$. An incompatible character made participants respond more slowly than did a compatible character (by $14 \mathrm{msec}$ ). When a distractor cue was present, this compatibility effect disappeared $[t(9)=1.59, p=.147]$. However, in this case, there was a significant compatibility effect with the

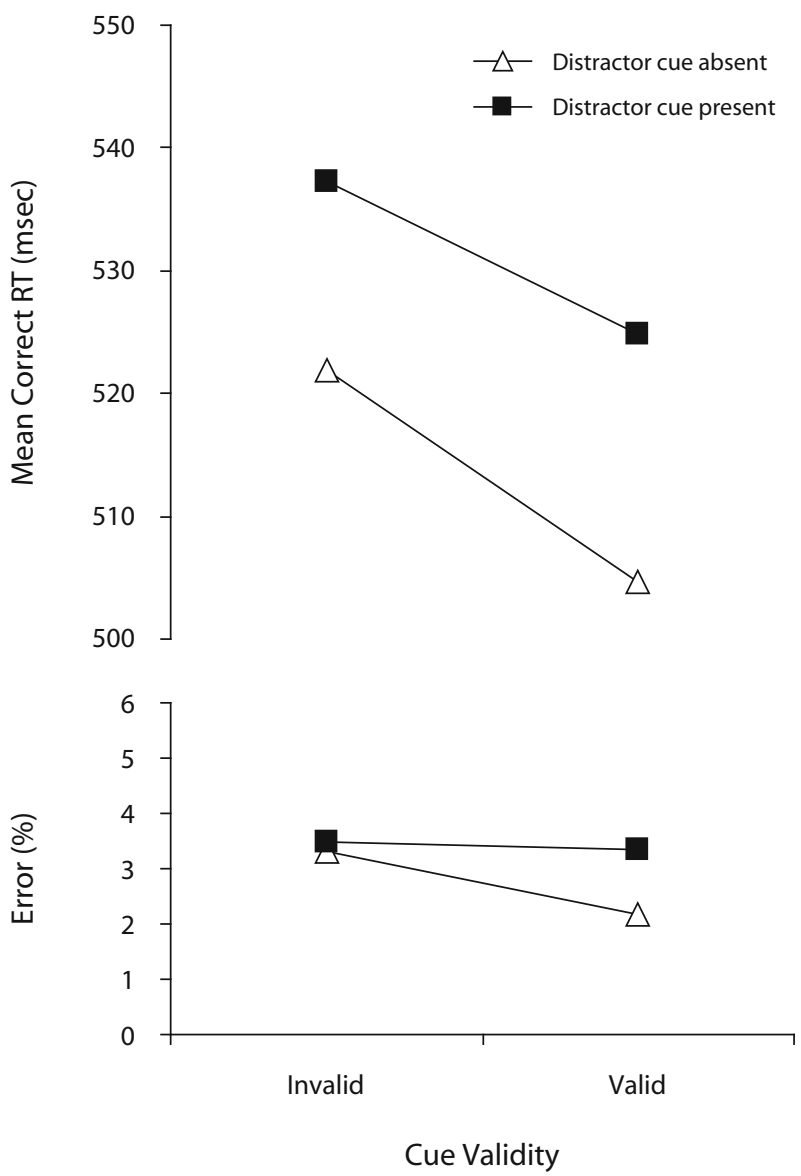

Figure 2. Results of Experiment 1. The response times (RTs) and error percentages as a function of cue validity, in the situations where a second red distractor was absent or present. character at the red distractor location $[t(9)=2.87, p<$ .05 (by $14 \mathrm{msec}$ )]. Thus, the identity of the character at the distractor cue interfered with the response to the target.

An analysis of the errors with similar factors yielded no surprises. Error rates were equal among all conditions and levels.

The contingent capture account leaves us no other option than to assume that the red distractor captured attention, since (1) its color matched the participant's attention set for the task, and (2) it resulted in increased RTs. If one denied that the red distractor captures attention, one would run the risk of also having to deny that the red cue captures attention, which obviously goes against the claims of contingent capture. Even so, we find almost perfect additivity between the red cue and the red distractor. An invalid cue added about $20 \mathrm{msec}$ to RTs, as did the presence of a red distractor, regardless of cue validity.

The costs inflicted by the red distractor in this experiment were generally of greater magnitude than were the costs inflicted by an onset distractor in Schreij et al. (2008). Most likely, this is because the red distractor contained a task-relevant feature, which may have resulted in more trouble in disengaging attention from the distractor. The observation was made that the cue-induced compatibility effects also corroborate the claim that the distractor successfully captured attention away from the first cue, since there is only a compatibility effect of the first cue when the distractor cue is absent and, when present, the distractor cue yields its own compatibility effect. Yet, despite these somewhat stronger distractor effects, the relationship with the cuing effect remained additive.

It is also unlikely that the costs generated by the distractor cue are due to filtering, since, according to the filter account, filtering costs generally only accompany the appearance of a new perceptual object, and the distractor cue was actually formed by a feature change to an old, already present object. In addition, this change occurred $100 \mathrm{msec}$ before the target appearance, whereas filtering costs are claimed to be usually manifested only with an appearance simultaneous with the target (Folk \& Remington, 1998; Kahneman et al., 1983).

To conclude, it appears that the onset effects, as found in Schreij et al. (2008), are very similar to contingent capture effects, in terms of their additivity with earlier capture effects. It seems that contingent capture is partly a sustained phenomenon whose lingering effects are not easily terminated by subsequent events. It is possible that such sustained effects are due to the seemingly task-relevant nature of the capturing event, which may trigger slower but longer lasting top-down feedback mechanisms. If underadditivity is a strict prerequisite for attentional capture by the onset distractor in previous experiments, contingent capture by the red distractor in the present experiment should not have been additive to the effect of the color cue; in other words, if we accept that the distractor captured attention in the present experiment, additivity per se is not a diagnostic for or against attentional capture. Thus, the argument that additivity between cue validity and onset presence is better explained by filtering costs, and thus excludes attentional capture, is doubtful. 


\section{EXPERIMENT 2}

The important question remains why later distractors (whether color based or onset based) did not obliterate the earlier cuing effect (i.e., cause an underadditive interaction of cuing and distractor effects). We have already suggested that the attentional priority induced by the cue may linger, thus exerting effects even after observers have already visited the next distractor. This would still mean that if there were a distractor powerful enough to capture attention away from the location of the contingent precue, then hold attention long enough for the cuing effect to finally dissipate, underadditivity might be observed. With a 10 - to $20-\mathrm{msec}$ interference effect, the onset distractor in Schreij et al. (2008), or the attentional set-matching red distractor of Experiment 1, may not have been strong enough. We expected that, if anything, a distractor both salient in a stimulus-driven fashion (i.e., featuring an abrupt onset) and carrying a feature also possessed by the target at the same time (i.e., its bounding box was red) might do the job, since it would evoke both contingent top-down and stimulus-driven attentional resources. It has been argued before that attentional disengagement from items possessing task-relevant features is more time consuming than such disengagement from irrelevant items (Theeuwes et al., 2000). We argued that, if the onset distractor manages to occupy attentional resources long enough, any residual effects of the precue will have dissipated once attention has been able to disengage from the distractor location. The consequence would be that attention will not return to the previous position of this cue, eliminating the cuing effects found in previous experiments, this time resulting in an underadditive relation between onset presence and cue validity. At the same time, this would again demonstrate a crucial contribution of the onset to attentional capture.

\section{Method}

Participants. Thirteen students at Vrije Universiteit Amsterdam, age range 18-30 (average 24), participated in this experiment in return for money or course credits. All reported no color blindness, and normal or corrected-to-normal vision.

Apparatus, Stimuli, Design, and Procedure. The apparatus and stimuli were largely similar to those in Experiment 1, except that there was no longer a distractor cue and that, in a new contingent onset distractor condition, the distractor was a white onset character appearing in a red bounding box positioned between two old bounding boxes (in addition to the irrelevant onset condition). The target itself never appeared through an abrupt onset. In addition, the ISI between the cue and target display was reduced from $150 \mathrm{msec}$ (as in Experiment 1) to $100 \mathrm{msec}$, as it was in Folk et al. (1992). Thus, the important factors of this experiment were cue validity (invalid, valid) and onset presence (no onset, onset), which were varied within blocks, and onset distractor type (irrelevant onset, contingent onset), which was varied between blocks. The experiment consisted of 2 practice blocks and 10 experimental blocks, each consisting of 80 trials, which took participants around $45 \mathrm{~min}$ to complete.

\section{Results and Discussion}

Incorrect responses were removed from the data set, discarding $3.7 \%$ of the trials, as were responses with RTs below and above $2.5 S D$ from the mean (another 2.1\%).
The remaining data are depicted in Figure 3 and were submitted to an ANOVA with cue validity (valid, invalid) and onset presence (no onset, onset) for both conditions of onset distractor type (contingent vs. irrelevant) as factors.

When the onset had an irrelevant white bounding box, cue validity and onset presence were significant as before $[F(1,12)=17.66, p<.001$, and $F(1,12)=13.69, p<$ .001 , respectively], demonstrating that a valid cue yielded considerable response benefits and that the presence of an onset slowed down participants. The interaction between these two factors was far from significant $(p>$.6). In the blocks where the onset had a red bounding box, there was no main effect of cue validity $[F(1,12)=1.07, p=.321]$, indicating no difference between RTs for a valid and an invalid cue. The main effect of onset presence was significant $[F(1,12)=13.08, p<.001]$. Participants' responses were delayed when an onset was present. Importantly, the interaction between cue validity and onset presence was significant $[F(1,12)=6.59, p<.05]$. When present, a contingent red onset annihilated the validity effect of the earlier color cue. Pairwise comparisons of the valid and invalid cue conditions revealed an effect of the cue in the noonset condition $[t(12)=2.379, p<.05]$, but not when a contingent onset was present $[t(12)=-0.471, p=.646]$.

A comparison of the no-onset trials in the contingent onset blocks with the irrelevant onset blocks showed that participants were significantly slower during the contingent onset blocks $[F(1,12)=5.62, p<.05]$. Conducting an ANOVA with onset distractor type (contingent, irrelevant) and cue validity (invalid, valid) as factors on all onset-present trials revealed that participants were significantly slower when a contingent red onset was present than when an irrelevant onset was present $[F(1,12)=$ $10.21, p<.001]$. The interaction between cue validity and onset presence was significant as well $[F(1,12)=8.52$, $p<.001]$, showing that the cuing effect got attenuated by the contingent red onset distractor.

For completeness, we also investigated whether the onset character caused a response compatibility effect with the target for both levels of onset distractor type. An ANOVA with cue validity (invalid, valid) and compatibility (incompatible, compatible) as factors revealed a significant main effect for compatibility in both irrelevant and contingent onset conditions $[F(1,12)=32.597, p<.01$, and $F(1,12)=6.708, p<.05$, respectively]. Participants thus responded slower with an incompatible onset than with a compatible one, whether or not it contained taskrelevant features. In neither onset distractor type condition was there an interaction of compatibility and cue validity ( $p>.1$ for both conditions).

Analysis of the error rates in the irrelevant onset blocks revealed no significant effects. In the contingent onset blocks, only onset presence reached significance $[F(1,12)=82.94, p<.001]$. Participants made significantly more errors when a contingent red onset was present than when it was absent.

Thus, distractors matching the participants' attentional set inflicted larger RT costs and were accompanied by 
A

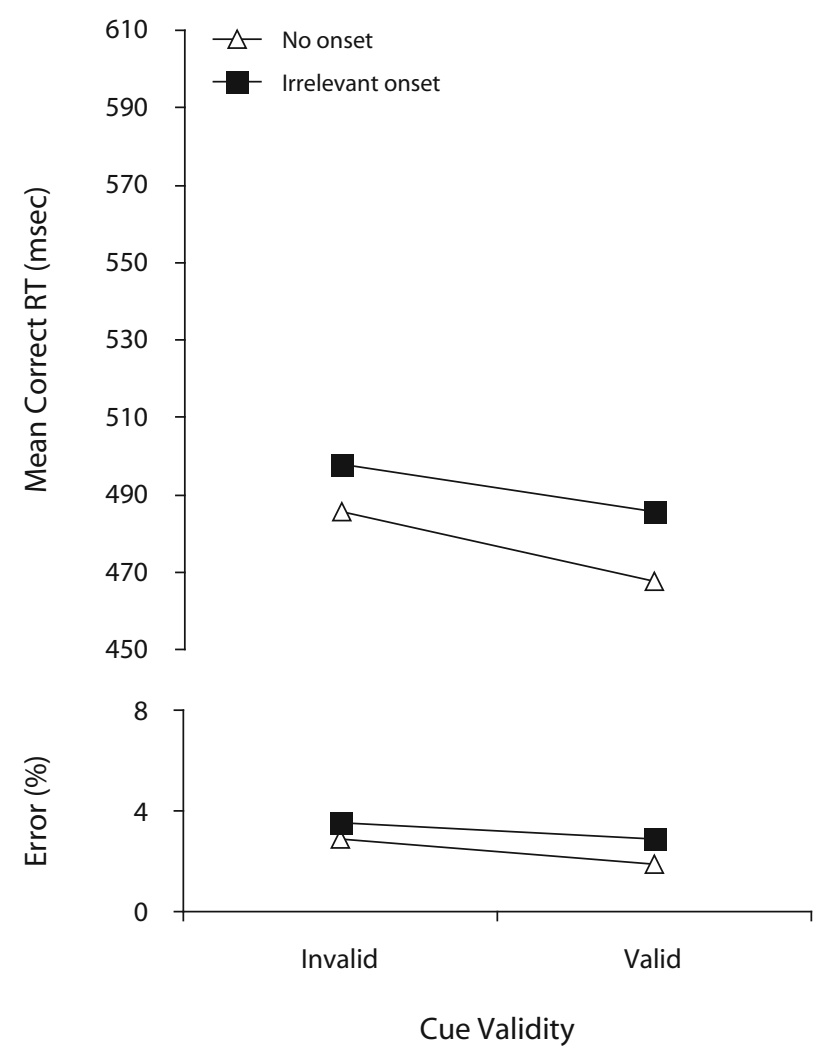

B
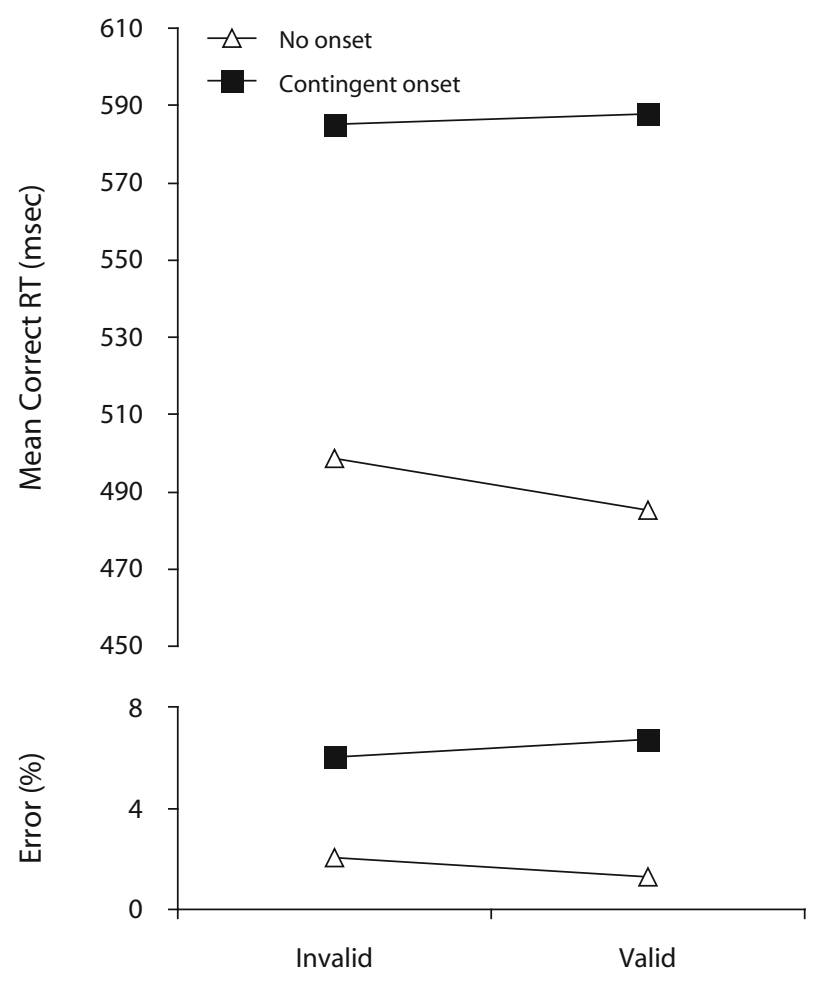

Cue Validity

Figure 3. Results of Experiment 2. The response times (RTs) and error percentages as a function of cue validity, in situations where there was no onset and where the onset appeared in (A) a white box (normal onset) or (B) a red box (contingent onset).

larger error rates than were distractors that were not contingent on the attentional set. This supports the notion that attentional capture toward and/or attentional disengagement from task-relevant items is more time consuming than for items deemed irrelevant (Theeuwes et al., 2000). Additionally, a contingent red onset distractor managed to completely eliminate the effect of the precue, as opposed to the onset-only distractor. Apparently, the combination of bottom-up salience and top-down task relevance gave the distractor the necessary boost to completely overrule a lingering cue validity effect.

Another interesting finding is that RTs were generally higher in contingent distractor blocks than in irrelevant distractor blocks, even on no-onset trials. Participants apparently became more conservative overall in responding when they knew that the distractor could share critical features with the target. This overall delay may also have allowed for further decay of lingering cuing effects.

The interaction between cue validity and the contingent red onset found in the present experiment exhibits the stronger notion of capture proposed by Folk et al. (2009), in that it meets the criterion of underadditivity. However, it also adds a constraint, in that the capturing item has to be sufficiently strong to overcome the earlier cuing effects. In the present case, adding a strong bottom-up signalan abrupt onset — was sufficient. If the distractor lacks a strong bottom-up signal (as in Experiment 1) or does not contain a task-relevant feature (as in Schreij et al., 2008), residual activation of a precue will be able to overcome capture by the irrelevant item and draw attention back to its location, with additivity as a consequence. In any case, the conclusion is that bottom-up signals strongly contribute to attentional capture.

\section{EXPERIMENT 3}

If the filtering account is correct, onsets do not capture attention when the observer is not looking for onsets. If anything, the observer is set against onsets, since they need to be filtered out from competition in order to prevent interference with target processing. The prediction, then, is that occasional targets featuring an abrupt onset should not be processed any faster than normal targets that feature no abrupt onset. In contrast, if onsets automatically draw attention, as stated by the capture account, a target appearing with an abrupt onset should allow for faster responses than do no-onset targets.

Earlier work by Yantis and Jonides (1984) has indeed shown that onset targets are given priority, even when the abrupt onsets are distractors on the majority of trials. In their experiments, observers viewed a display of premasks, one of which was likely to change into a target. 
Together with the display change, a new object, which only occasionally was the target, was added to the display. Nevertheless, search was faster and more efficient when the new object indeed turned out to be the target, consistent with the idea that it captured attention (see also Becker, 2007). Here, we wished to apply exactly the same logic to the paradigm used by Folk et al. (1992). In the present experiment, the onset coincided with a distractor on the vast majority of trials (as in the previous experiments), but now it could also accidentally coincide with the target (again a red item); hence, observers had no incentive whatsoever to attend to the abrupt onset and would be expected to employ an attentional set only for red (as indicated once more by a cuing effect). If the filtering account is correct and the onset does not capture attention, there should be no benefit for onset over noonset targets. If the capture account is correct-following Yantis and Jonides (1984) — response benefits should be observed for onset targets, because onsets involuntarily capture attention.

\section{Method}

Participants. Eight students from Vrije Universiteit Amsterdam, age range 20-30 (average 24), participated in a half-hour session in exchange for course credit or money.

Apparatus and Stimuli. Apparatus and stimuli were largely the same as in Experiment 2, with the exception of the following adaptations: In the onset distractor condition, an extra light gray bounding box containing a bright white character (either "X" or "=") suddenly appeared in the search display at the same moment that the other characters were revealed. In the onset target condition, this character was presented in red.

Design and Procedure. The design and procedure were mostly identical to those of Experiment 2, except for the following: There were no more contingent distractor blocks. A new factor, onset type, consisted of the following levels. In the no-onset condition, there was no additional onset present. In the onset distractor condition, the new onset contained a distractor item ("X" or "="). In the onset target condition, the new onset contained the target. This implied that in both the onset distractor and onset target conditions, the effective set size was 5 , whereas in the no-onset condition it was 4 . Cue validity was varied, as in Experiment 2, but note that in the onset target condition the cue was always invalid (since the empty location in which the onset target appeared could not be cued). There were eight blocks of 80 trials each, preceded by a practice block of 40 trials. Only 6 of the 80 trials per block contained an onset target. The onset was a distractor on 34 trials, and there was no onset on the remaining 40 trials.

\section{Results and Discussion}

Incorrect responses were removed from the data set, resulting in a loss of $3 \%$ of the trials, as were responses with RTs below and above $2.5 S D$ from the mean (another 2\%). The remaining data are shown in Figure 4 and were first submitted to an ANOVA with onset type (no-onset, onset distractor) and cue validity (valid, invalid) as factors. The onset target level was omitted in this analysis, because it could not be fully crossed with cue validity (since onset targets could only be invalidly cued; see the Method section). We will return to onset targets below. There was a significant main effect of cue validity $[F(1,7)=49.48$, $p<.001]$. Participants were slower after an invalid cue than after a valid cue. The presence of an onset distractor also slowed the responses [onset type, $F(1,7)=40.80$,
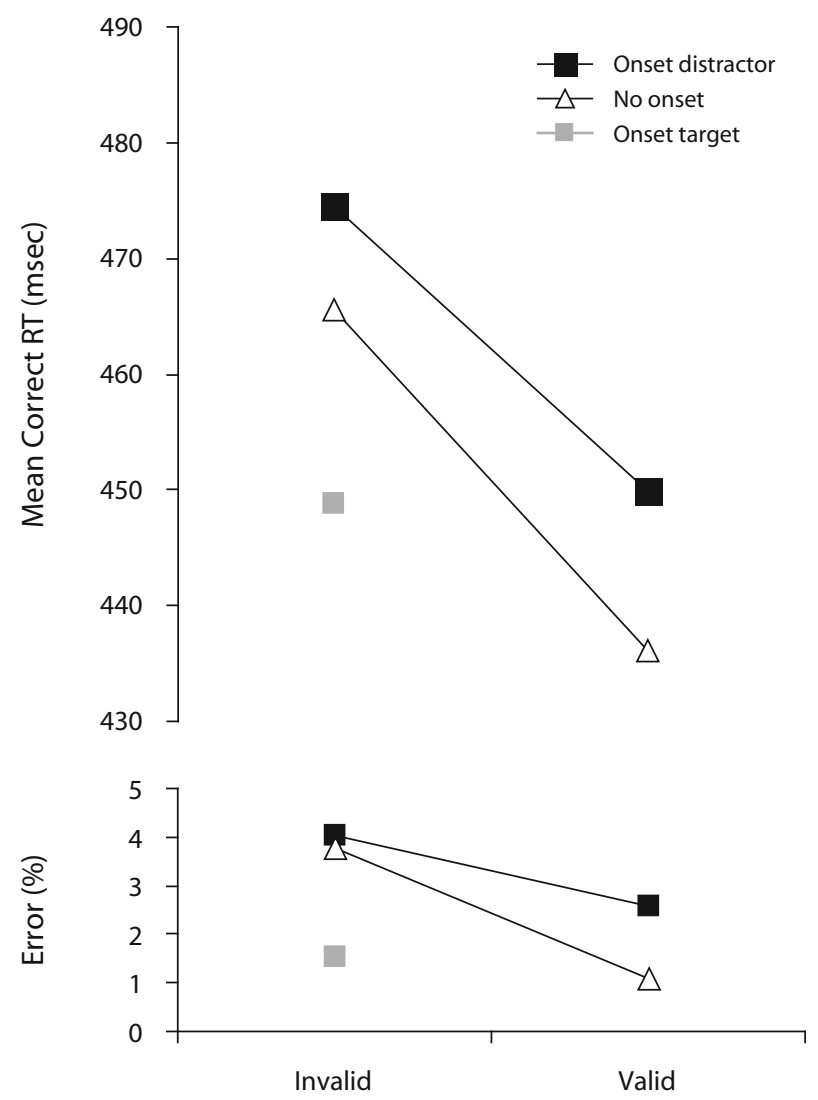

Cue Validity

Figure 4. Results of Experiment 3. The response times (RTs) and error percentages as a function of cue validity, when the onset was a target or a distractor, or when there was no onset at all.

$p<.001]$. There was no interaction between onset type and cue validity $[F(1,7)<1.9, p>.2]$, pointing toward additive effects.

To assess the effect of an onset target, we examined invalidly cued trials only for onset target, onset distractor, and no-onset trials. A one-way ANOVA revealed a main effect of onset type $[F(2,14)=26.05, p<.001]$. Separate comparisons revealed a significant benefit for onset target trials relative to no-onset and onset distractor trials $[t(7)=$ $4.13, p<.01$, and $t(7)=5.70, p=.001$, respectively]. RTs on onset distractor trials were significantly slower than on no-onset trials $[t(7)=5.65, p=.001]$.

Analysis for compatibility effects with cue validity (valid, invalid) and compatibility (incompatible, compatible) as factors once more revealed a significant effect of target-distractor compatibility $[F(1,7)=32.739, p<$ .001]. Participants responded slower to a target when it was accompanied by an incompatible rather than a compatible distractor. There was no interaction between cue validity and compatibility $(p>.8)$.

The error pattern largely followed that of the RTs, and the same analyses revealed no significant effects, although cue validity approached significance $[F(1,7)=$ $6.156, p=.056]$. 
The results clearly demonstrate a performance benefit over no-onset trials when the target featured an abrupt onset (in the invalid cue condition), as opposed to costs when the onset coincided with a distractor. This provides direct evidence for an attentional capture account: Despite its irrelevance to the task, the abrupt onset results in a local enhancement of processing, leading to benefits when it is a target and costs when it is a distractor. No such benefits would be predicted by the filtering account: According to this account, the onset is irrelevant. As the cue validity effects once more demonstrate, the observers were set for red, not for onsets. In defense of a filter account, one might argue that the transience of the onset made the target more salient, and therefore easier to orient to, but if this were the case, it would argue against the primary idea of contingent capture - namely, that the allocation of attention does not depend on salience, but only on task relevance. The idea that high salience facilitates (or invokes involuntary) orientation of attention toward objects is usually a claim made by the attentional capture account. Another possibility is that the onset benefit stems from differential masking caused by the premasks for old objects; however, we consider this unlikely. Using the present displays, Schreij et al. (2008, Experiments 1 and 2) directly compared performance with and without premasks and found no influence of this on the interference caused by abrupt onsets.

\section{GENERAL DISCUSSION}

There has been a long-standing discussion of whether irrelevant visual objects demand spatial attention or additional filtering operations (Becker, 2007; Folk \& Remington, 1998; Kahneman et al., 1983; Theeuwes, 1994; Theeuwes \& Burger, 1998). These two mechanisms are by no means mutually exclusive, and, as pointed out by Becker, different paradigms may result in a different emphasis on one or the other. This is why here and in Schreij et al. (2008), we chose to integrate several attentional capture paradigms (Folk et al., 1992; Theeuwes, 1992; Yantis \& Jonides, 1984) and see whether particular findings generalize. Experiments 1 and 2 investigated the claim made by Folk et al. (2009) that if a distractor captures attention away from the cued location, its effect should be underadditive with the effect of cue validity. This implies that if the onset distractor captures attention, there should be few to no response benefits for a valid preceding cue. Experiment 1 demonstrated that a contingent no-onset distractor was able to capture attention away from the precued location but that a target appearing at this location still enjoyed considerable response benefits. The contingent distractor effect was additive with the contingent cuing effect, suggesting that (1) the effects of cuing were prolonged well into the target display and (2) additivity per se is not a good diagnostic for or against attentional capture. Experiment 2 then showed that when an abrupt onset was added to the contingent distractor, the cuing effects disappeared. We infer that only a sufficiently strong distractor is capable of eliminating lingering cuing effects - in this case, when it has a strong bottom-up signal and is also contingent on the participants' attentional set. In Experiment 3 , onset costs turned into benefits when the onset coincided with the target location, indicating preferential processing of onsets. According to the useful taxonomy of capture and filter effects outlined by Becker, the latter effect especially meets the criterion for attentional capture: Prioritized processing of an item is not expected if the RT costs it generates when it is a distractor result from a filtering operation. Since a filtering operation is assumed to only suppress elements, a facilitated response to objects that should normally be filtered out is exactly the opposite of what one would expect, and can be better explained by attentional capture.

Another possible explanation for the priority given to the abrupt onset here and in Schreij et al. (2008) might be that here and in the previous experiment an onset distractor was disruptive because participants operated in "singleton detection mode" (Bacon \& Egeth, 1994) in a search for the target. Since the target was the only red character among white distractors, it constituted a color singleton, a property that participants might have actively used to find it. An onset, however, may also temporarily obtain a singleton status, due to its strong transient; therefore, the onset and color singleton might be direct competitors as the only salient elements in the display, when participants search for any salient object. However, Experiment 4 of Schreij et al. (2008) precludes this explanation. Even when the color target was not a singleton, the onset still interfered. In this case, all distractors were heterogeneously colored and participants were required to actively search for redness, forcing them to use "feature detection mode" (Bacon \& Egeth, 1994). Furthermore, the present setup closely matched that of the original Folk et al. (1992) experiments, in which the targets were also singletons, yet there was no evidence of singleton detection mode there, since singletons that did not match the attentional set did not result in cuing effects.

Although we believe that our findings support the claim that onsets have a special status of being able to capture attention regardless of one's attentional set, the results appear to contradict the findings of Folk et al. (1992), showing that an abrupt onset cue did not cause any validity effect (and hence did not appear to capture attention), when participants searched for a target defined by color. Theeuwes et al. (2000) explained this lack of capture in the Folk et al. (1992) paradigm by reasoning that, during the short SOA between cue and target appearance, any capture effect of an irrelevant cue might have already dissipated, especially since the irrelevant cue contained no task-relevant features. This made swift disengagement from the distractor location possible. In the Folk et al. (1992) study, the onset cue appeared $150 \mathrm{msec}$ prior to the target, whereas in most studies in which capture by irrelevant onsets was found, the onset appeared simultaneously with the target, leaving no time for the capturing power of the onset to diminish. After all, Schreij et al. (2008) found the costs inflicted by an onset distractor appearing simultaneously with the target to be only $15 \mathrm{msec}$ on average, which is a short enough time to have already passed in the SOA between cue and target used in the experiments of Folk et al. (1992). 
However, Lamy and colleagues (Lamy, 2005; Lamy \& Egeth, 2003, Experiments 3 and 4) have shown that an irrelevant onset cue was actually able to capture attention even when it appeared before the target, as in Folk et al.'s (1992) original experiments. These studies identified onset salience and the predictability of onset-to-target SOA as important factors for evoking involuntary capture by irrelevant onsets. An onset cue managed to capture attention when it was relatively salient and preceded the target at an unpredictable SOA. These parameters were fixed in the original Folk et al. (1992) study, and seemed important for modulating capture by onsets. Because the onset distractor was presented before the target display, the measured effects were unambiguously spatial. The fact that Theeuwes et al. (2000) found capture only for short SOAs, in spite of using an unpredictable distractorto-target SOA, could possibly be attributed to the fact that they used a less salient, static (color-based) distractor, instead of an onset distractor.

Related to the idea that the onset has to be sufficiently salient, previous research has shown that the capturing power of an onset is largest when it also makes up a new perceptual object (Enns et al., 2001; Yantis \& Hillstrom, 1994). The onset of a complete box including a letter, as used by us here, can be regarded as a new object, but this may not be true for the four small white dots that Folk et al. (1992) used as their onset precue. These might have been perceived as a property change of an old objectnamely, the bounding box that they surrounded. For this reason, the capturing power of the cue used in Folk et al. (1992) paradigms might not have been sufficient for longstanding effects, this being the reason that they found a lack of capture by an onset cue when participants searched for a color target; but see Folk and Remington (1999) for a study of contingent capture with new-object onsets.

Further support for the notion that new object onsets capture attention comes from research by Brockmole and Henderson (2005). In a study, they tracked eye movements over natural scenes; a new object was abruptly presented either during fixation or during a saccade. In both situations, the new object was fixated more often than chance. However, onsets that appeared during fixation were fixated sooner and more often than those coinciding with saccades. This made the authors conclude that a new object does not need to have a perceivable transient to capture the eyes; a nontransient new object is capable of doing so as well, though to a lesser extent. The fact that these effects were not modulated by observers' expectations concerning the appearance of new objects strengthens the notion that onset prioritization is involuntary, and the finding that our eyes consistently move to new objects underpins the notion that this prioritization is spatial in nature (see also Theeuwes, Kramer, Hahn, \& Irwin, 1998).

\section{Identity Intrusion}

Schreij et al. (2008) found additional evidence for attentional capture by the onset in an experiment using the so-called identity intrusion technique first introduced by Theeuwes (1996; see also Theeuwes \& Burger, 1998). Instead of presenting a neutral abrupt onset, the abrupt onset was either compatible or incompatible with the response to the identity of the target. This technique rests on the assumption that if attention is shifted to the location of the abrupt onset, its identity will be preferentially processed (e.g., Kramer \& Jacobson, 1991). An incompatible distractor identity would then more greatly degrade task performance than would a compatible one. Consistent with this, Schreij et al. (2008) found a response compatibility effect.

Folk et al. (2009), however, have argued that this response compatibility effect can also be explained by parallel processing of the target and the distractor. In support of this argument, they conducted an experiment in which participants were presented with a display with two boxshaped placeholders, on the left and right sides of fixation, in which two characters appeared. One of these characters was a red target; the other was a white distractor, which could be either compatible or incompatible with the target. Prior to the target display, one of the positions was indicated by an uninformative red cue. They found that even when the target was correctly cued, at which point attention should be focused on the target location, the compatibility of the distractor still affected RTs, despite the fact, as argued by Folk et al. (2009), that this distractor did not appear as an abrupt new onset. Since participants had no reason whatsoever to attend to the irrelevant character when the cue was valid, but nonetheless registered its identity, the conclusion was that both characters were processed in parallel, contrary to what a spatial attentional capture account would predict.

Note that this explanation assumes that filtering is not always perfect. If the distractor is indeed processed in parallel with the target to the extent that it directly interferes with the response to that target, the distractor was not filtered out successfully. If filtering were successful, there would be no interference (what would otherwise be the purpose of filtering?). But just as filtering may not always be perfect, participants may also not always perform perfectly in attending to the cue; in other words, attention may not always be perfectly focused on the target, as assumed by Folk et al. (2009) in their experiment. Observers may occasionally accidentally attend to the distractor, because when it appears it shows dynamic change similar to that shown by the target. The two stimuli might even group on the basis of such common dynamics. All such factors might contribute to a response compatibility effect.

\section{Additivity}

As in Schreij et al. (2008), we found that the contingent cuing effects and the standard onset distractor effect were additive. Folk et al. (2009) argued that the additivity of the onset distractor effects and the spatial cuing effects meant that they could not both operate on the same level of spatial attention. If we accept that contingent cuing affects spatial orienting, onset capture cannot do so too. However, we have arguments against this claim. It is conceivable that activation at a cued location is higher and more sustained over time because the cue contains a task-relevant feature. For this reason, attention might "snap back" to the cued location, after the abrupt onset in the subsequent tar- 
get display was briefly able to attract attention away from there. The short excursion of attention causes an additive effect. Folk et al. (2009) argue against this "rubber band" explanation on the ground that the cue has already disappeared after the target display and onset are presented, and thus should no longer be able to attract attention. If anything, attention should be repelled by the old location, due to the mechanism known as inhibition of return (IOR; Posner \& Cohen, 1984).

However, we believe that it would not be the first time that an already disappeared stimulus still exerted considerable effects in the cognitive system. For one, the (red) cue is not masked, so any cue-related activity is expected to automatically linger for a while. Moreover, this activity is likely to be maintained, or even strengthened, by merit of its being relevant to the observer (who, after all, is looking for something red). IOR is then quite unlikely, because (1) the cue is considered relevant by the system (IOR is found to be weak under these conditions; Pratt, Sekuler, \& McAuliffe, 2001), and (2) the time between cue and target display is only $150 \mathrm{msec}$, which is typically too short to generate IOR (Posner \& Cohen, 1984).

Furthermore, the cue may already have triggered higher order processes involving working memory and nonspecific response preparation, processes not easily disengaged or wiped out by the presentation of the onset distractor and that may demand that resources return to the original location. In fact, Theeuwes and Godijn (2002) conducted an eye-movement study that directly supports the idea of sustained activation for locations of interest in the visual field, even when the stimulus has disappeared. Participants searched for a color singleton in a circular array of disks while eye movements were recorded. On some of the trials, an irrelevant onset distractor appeared that often captured the participants' gaze. Importantly for the present discussion, during the eye movement to the onset distractor the target color singleton had moved to another location. Nevertheless, on $82 \%$ of the trials, the participants' next eye movement was toward the old target location, not the (by now more salient) new target location.

Even if we accept that there must be two different stages involved, can one really claim that contingent cuing effects reflect only spatial capture and no other processes? For example, the disengagement times from invalid cues found by Folk et al. (1992) are of a much greater magnitude than the ones usually found for items assumed to exogenously have captured attention (Theeuwes et al., 2000). As has been argued by Theeuwes et al. (2000), top-down effects on attention, such as those found in the contingent capture paradigm, may at least partly involve disengagement and/ or decision mechanisms, rather than just initial orienting. Such effects may well turn out to be considered additive with the spatial capture effects caused by an abrupt onset, thus reversing the argument.

\section{CONCLUSION}

It is clear that many questions remain about the exact mechanisms of attentional capture, and that more sophisticated paradigms, as well as better definitions, are nec- essary. What we have argued here is that abrupt onsets summon attention, despite observers' employing an attentional set for a certain color. We do not deny a role for top-down influences on attentional capture, but here we show again that some stimuli are, to a certain extent, immune to this top-down set.

\section{AUTHOR NOTE}

This work was supported by VIDI Grant 452-06-007 from the Netherlands Organization for Scientific Research (NWO) granted to C.N.L.O. We thank Chip Folk, Jim Brockmole, and Dominique Lamy for their constructive reviews and suggestions. Correspondence concerning this article should be addressed to D. Schreij, Department of Cognitive Psychology, Vrije Universiteit, Van der Boechorststraat 1, 1081 BT Amsterdam, The Netherlands (e-mail: dbb.schreij@psy.vu.nl).

\section{REFERENCES}

BACON, W. F., \& EgETh, H. E. (1994). Overriding stimulus-driven attentional capture. Perception \& Psychophysics, 55, 485-496.

BECKER, S. I. (2007). Irrelevant singletons in pop-out search: Attentional capture or filtering costs? Journal of Experimental Psychology: Human Perception \& Performance, 33, 764-787.

Belopolsky, A. V., Schreis, D., \& Theeuwes, J. (2010). What is topdown about contingent capture? Attention, Perception, \& Psychophysics, 72, 326-341.

Belopolsky, A. V., Theeuwes, J., \& Kramer, A. F. (2005). Prioritization by transients in visual search. Psychonomic Bulletin \& Review, 12, 93-99.

Brockmole, J. R., \& Henderson, J. M. (2005). Prioritization of new objects in real-world scenes: Evidence from eye movements. Journal of Experimental Psychology: Human Perception \& Performance, 31, 857-868.

Christ, S. E., \& Abrams, R. A. (2008). The attentional influence of new objects and new motion. Journal of Vision, 8(3, Art. 27), 1-8.

Enns, J. T., Austen, E. L., Di Lollo, V., Rauschenberger, R., \& YANTIS, S. (2001). New objects dominate luminance transients in setting attentional priority. Journal of Experimental Psychology: Human Perception \& Performance, 27, 1287-1302.

Folk, C. L., Leber, A. B., \& Egeth, H. E. (2002). Made you blink! Contingent attentional capture produces a spatial blink. Perception \& Psychophysics, 64, 741-753.

Folk, C. L., \& Remington, R. (1998). Selectivity in distraction by irrelevant featural singletons: Evidence for two forms of attentional capture. Journal of Experimental Psychology: Human Perception \& Performance, 24, 847-858.

Folk, C. L., \& Remington, R. (1999). Can new objects override attentional control settings? Perception \& Psychophysics, 61, 727-739.

Folk, C. L., Remington, R. W., \& Johnston, J. C. (1992). Involuntary covert orienting is contingent on attentional control settings. Journal of Experimental Psychology: Human Perception \& Performance, 18, 1030-1044.

Folk, C. L., Remington, R. W., \& Wu, S. C. (2009). Additivity of abrupt onset effects supports nonspatial distraction, not the capture of spatial attention. Attention, Perception, \& Psychophysics, 71, 308-313.

Gellatly, A. (1999). Perception and information processing. Behavioral \& Brain Sciences, 22, 377-378.

Kahneman, D., Treisman, A., \& Burkell, J. (1983). The cost of visual filtering. Journal of Experimental Psychology: Human Perception \& Performance, 9, 510-522.

Kramer, A. F., \& Jacobson, A. (1991). Perceptual organization and focused attention: The role of objects and proximity in visual processing. Perception \& Psychophysics, 50, 267-284.

LAMY, D. (2005). Temporal expectations modulate attentional capture. Psychonomic Bulletin \& Review, 12, 1112-1119.

Lamy, D., \& Egeth, H. E. (2003). Attentional capture in singletondetection and feature-search modes. Journal of Experimental Psychology: Human Perception \& Performance, 29, 1003-1020.

Posner, M. I., \& CoHEN, Y. (1984). Components of visual orienting. In H. Bouma \& D. G. Bouwhuis (Eds.), Attention and performance X: Control of language processes (pp. 531-556). Hillsdale, NJ: Erlbaum. 
Pratt, J., Sekuler, A. B., \& McAuliffe, J. (2001). The role of attentional set on attentional cuing and inhibition of return. Visual Cognition, 8, 33-46.

Remington, R., Johnston, J. C., \& Yantis, S. (1986). Do abrupt onsets capture attention involuntarily? Bulletin of the Psychonomic Society, 24, 347.

SchreiJ, D., Owens, C., \& Theeuwes, J. (2008). Abrupt onsets capture attention independent of top-down control settings. Perception \& Psychophysics, 70, 208-218.

STERnBERG, S. (1969). The discovery of processing stages: Extensions of Donders' method. Acta Psychologica, 30, 276-315.

TheEUWES, J. (1990). Perceptual selectivity is task dependent: Evidence from selective search. Acta Psychologica, 74, 81-99.

Theeuwes, J. (1992). Perceptual selectivity for color and form. Perception \& Psychophysics, 51, 599-606.

Theeuwes, J. (1994). Stimulus-driven capture and attentional set: Selective search for color and visual abrupt onsets. Journal of Experimental Psychology: Human Perception \& Performance, 20, 799-806.

Theeuwes, J. (1996). Perceptual selectivity for color and form: On the nature of the interference effect. In A. F. Kramer, M. G. H. Coles, \& G. D. Logan (Eds.), Converging operations in the study of visual selective attention (pp. 297-314). Washington, DC: American Psychological Association.
Theeuwes, J. (2000). Attentional capture and oculomotor control. Perception, 29(ECVP Abstract Supp.), 2.

Theeuwes, J., Atchley, P., \& Kramer, A. F. (2000). On the time course of top-down and bottom-up control of visual attention. In S. Monsell \& J. Driver (Eds.), Control of cognitive processes: Attention and performance XVIII (pp. 105-125). Cambridge, MA: MIT Press.

Theeuwes, J., \& Burger, R. (1998). Attentional control during visual search: The effect of irrelevant singletons. Journal of Experimental Psychology: Human Perception \& Performance, 24, 1342-1353.

Theeuwes, J., \& GodiJn, R. (2002). Irrelevant singletons capture attention: Evidence from inhibition of return. Perception \& Psychophysics, 64, 764-770.

Theeuwes, J., Kramer, A. F., Hahn, S., \& Irwin, D. E. (1998). Our eyes do not always go where we want them to go: Capture of the eyes by new objects. Psychological Science, 9, 379-385.

Yantis, S., \& Hillstrom, A. P. (1994). Stimulus-driven attentional capture: Evidence from equiluminant visual objects. Journal of Experimental Psychology: Human Perception \& Performance, 20, 95-107.

YANTIS, S., \& Jonides, J. (1984). Abrupt visual onsets and selective attention: Evidence from visual search. Journal of Experimental Psychology: Human Perception \& Performance, 10, 601-621.

(Manuscript received October 31, 2008; revision accepted for publication October 31, 2009.) 